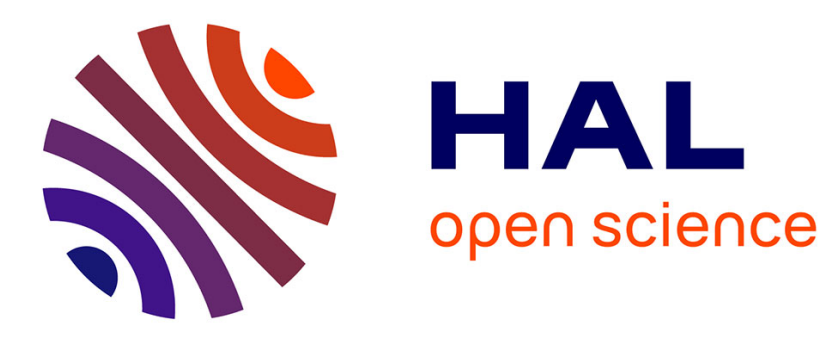

\title{
Découverte de poterie peinte à Toulon-sur-Allier (Allier) Hugues Vertet
}

\section{To cite this version:}

Hugues Vertet. Découverte de poterie peinte à Toulon-sur-Allier (Allier). Gallia - Fouilles et monuments archéologiques en France métropolitaine, 1959, 17 (2), pp.216-223. 10.3406/galia.1959.2266 . hal-01924475

\section{HAL Id: hal-01924475 \\ https://hal.science/hal-01924475}

Submitted on 3 Mar 2020

HAL is a multi-disciplinary open access archive for the deposit and dissemination of scientific research documents, whether they are published or not. The documents may come from teaching and research institutions in France or abroad, or from public or private research centers.
L'archive ouverte pluridisciplinaire HAL, est destinée au dépôt et à la diffusion de documents scientifiques de niveau recherche, publiés ou non, émanant des établissements d'enseignement et de recherche français ou étrangers, des laboratoires publics ou privés.

\section{(이) $\$$}

Distributed under a Creative Commons Attribution - NonCommercial - NoDerivatives| 4.0 


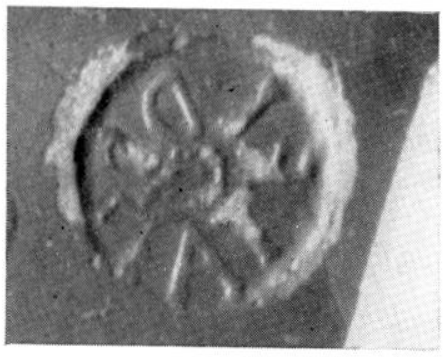

Fig. 3. -- L'estampille, agrandie. (Pholo H. Verlel).

La pelile estampille circulaire placée dans le fond intérieur de la panse permet de lire très facilement le génitif : $\triangle T E I$, mais l'aulre nom est dillicile à interpréter (fig. 1, 3). La première lettre est certaine : D. Mais nous n'avons trouvé au C.I.L. aucun nom d'esclave commençant par I), ni d'Aleius ni d'un autre potier de l'école arrétine. Sans doute avons-nous ici une nouvelle estampille et un nouveau nom.

Outre l'intérêt d'un décor complet et, d'une nouvelle signature, ce cratère apporte donc un élément intéressant au problème des rapports Bargathes (A)Aleius. S'il est vraisemblable que le moule de cette panse a été réalisé par le premier, l'estampille sur le fond du vase terminé mentionne un esclave du second. On connaît au moins un autre vase de cette sorte : c'est une belle pièce signée EROS ATEI, à Rome, dont les reliefs sont caractéristiques aussi de la fagon de Bargalhes Maître $\mathrm{A}^{13}$.

Nous sommes mal renseignés sur la vie de cet artiste décorateur qui travailla sûrement chez Perennius, dont il ajouta le génitif à son nom. Ce dernier vendit-il des moules de son ouvrier? prêta-t-il son esclave a l'oflicine d'Ateius? Y eut-il déplacement définitif de main d'œuvre? Ce problème, posé et étudié depuis longtemps déjà, ne semble pas avoir reçu encore de réponse définilive ${ }^{14}$.

Hugues VerTet.

quera une palmette identique à la palmette $B$ de la corbeille du vase de Périgueux. Dans l'étude que nous renons de citer, l'auleur remarque la préférence de Bargalhes Maître A pour la palmette qu'il dispose en variantes nombreuses (p. 46); sur le vase que nous venons d'étudier, on compte trois palmette différentes.

(13) DragendorfF-Watzinger, o.c., p. 51.

(14) Lal poterie d'Aleius se rattache à celle des débuts de Bargathes $A$ (qui n'est qu'une continuation de l'industric de Tigranus)

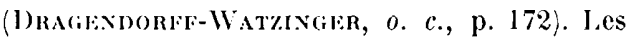
rapports rqui unissent à Cornelius et à Aleius Bargalhes a parlent en faveur d'une période qui serait la fin du régne d'Auguste et le début de celui de Tibere. (ibid., p. 54).

\section{I) Écoliverte de poterie peinte a Toulon-slr-Aldier (Aldier)}

En septembre 1957 , une campagne de fouilles conduite sur l'emplacement de l'atelier des potiers gallo-romains de Toulon-sur-Allier ${ }^{1}$ donna de très nombreux fragments des produits de cette oflicine. Mêlée à la céramique sigillée, se trouvait une céramique peinte repré-

(1) Plan cadastral, section I, parcelle n' 120 , lisière Sud. sentée sultisamment pour être classée parmi les productions de la fabrique. lille a été à peine mentionnée par les précédents fouilleur's, allirés surlout par les staluettes en terre blanche qui ont rendu Toulon célébre².

(2) Bullelin de la Sociélé d'émulation de l'Allier, VI, 1857 , p. 30 : «quelques fragments de poterie présentent même des rayures, des quadrillés de 


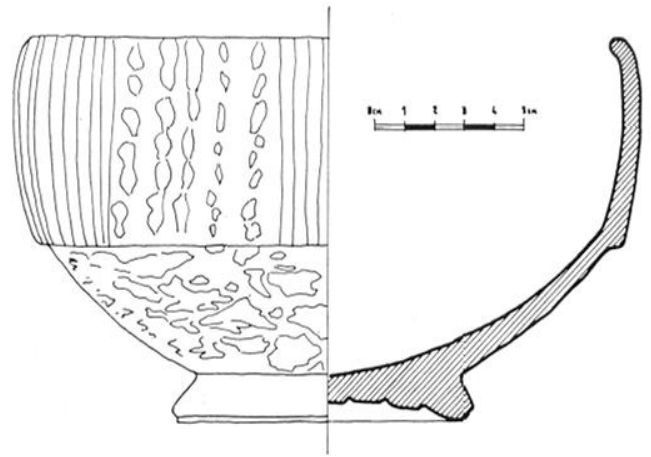

Fig. 1. - Forme de vase peint trouvé à Toulonsur-Allier. Ie fond a la couleur d'un mélange tres clair d'ocre orange, d'ocre jaune et de blane, et les parties peintes d'un mélange d'ocre orange et d'ocre jaune.

(Dessins II. Verlel)

Fig. 2. - Le haul en bas : 1. différents profils de rebords ; - - 2. profil de pied, inspiré des formes gallo-romaines (1 seul exemplaire trouvé) ; 3,4 et 5. trois formes de pied courantes.

Ce sont des bols tournés et lissés, dont le diamètre est voisin de 20 centimetres ${ }^{3}$. Forme et décors sont bien caractéristiques (v. fig. 1 et 2). Le rebord vertical, trìs élevé par rapport à la panse, varie de 4 centimètres it 7 cenlimètres do haut ${ }^{4}$.

nuances diverses "; PAYAN-I) umoutri, Anliquiles gallo-romaines decouverles à Toulon-sur-Allier, l.e Puy, 1860, sous le titre lases en lerre grise grossière: "Quelques vases ont des rayures de nuances variées qui paraissent avoir été peintes avec un vernis après la confection du vase" (p. 48).

(3) Diamètres calculés d'après les fragments de rebords et mesurés en centimètres : 16,6 ; 18,$8 ; 19,5 ; 19,6 ; 21,2 ; 22 ; 22,7 ; 22,8 ; 22,9$.

(4) Ilauteur de quelques rebords, en centimetres : 5,7 (signé OPPVI.), 7,4 (signé ALB JNIS:Épaisseur d̀ mi-hauteur en millimètres : ? (1 exemplaire), 3 (2 ex.), 4 (2 (x), 5 (4 ex.) et 6 ( 2 ex.).
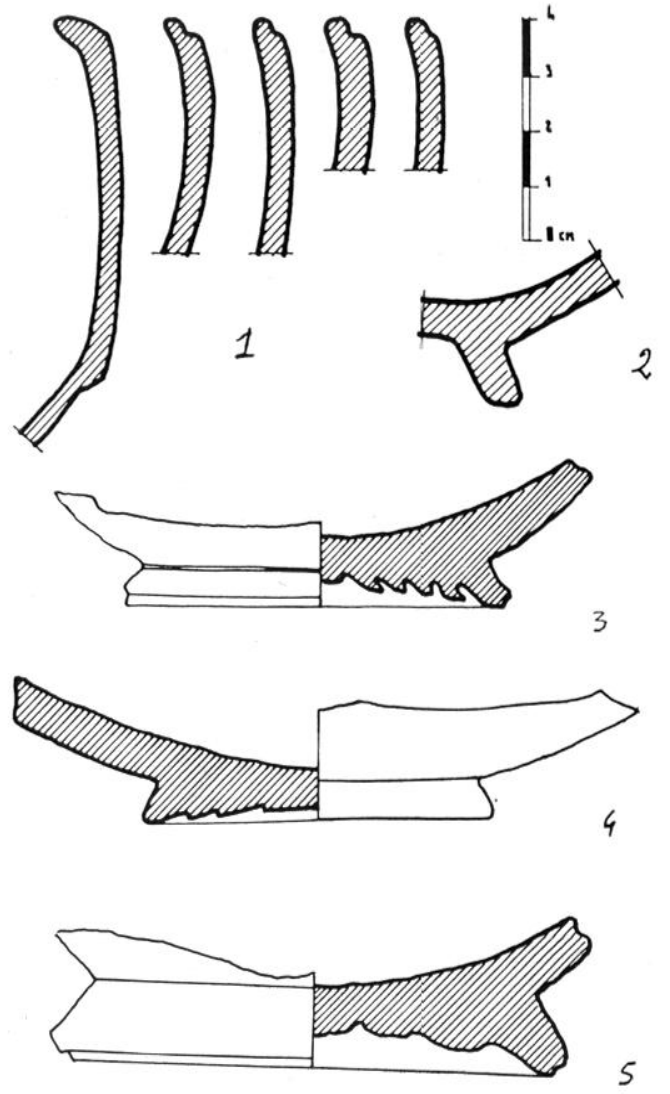

Sa levvre est toujours légèrement rentrante en haut, où elle se divise souvent en deux moulures, l'extérieure étant la plus basse (fig. $2, \mathrm{n}^{0} 1$ ). Très net, un décrochement extérieur ${ }^{5}$ le sépare de la panse courte et arrondie. A l'intérieur, il est marqué par un infléchissement de la paroi vers le centre du vase. Le bol est souvent brisé à celte hauteur, où il est plus mince. Le pied, légèrement évidé, tourné avec le vase, présente des stries circulaires au nombre de 2 à 5 , séparées par des surfaces bien lissées ou des bavures. Sur trente pieds trouvés, un seul a un profil différent ${ }^{6}$ (fig. $2, n^{\circ} 2$ ).

(5) Décrochements en millimètres : 3 (3 ex.), 4 (3 ex.), 5,5 ( 1 ex.), $6(1$ ex.). Epaisseur de la panse en millimètres : $5,7,8,9$ et 11 .

(6) Diamètres de quelques pieds en centi- 


\begin{tabular}{|c|c|c|c|c|}
\hline NOMS DES POTIERS & $\begin{array}{c}\text { NOMBRE } \\
\text { de } \\
\text { SIGNATURES }\end{array}$ & $\begin{array}{c}\text { FORME } \\
\text { DE LEUR SIGNATURE }\end{array}$ & DATE D'OSWALD ${ }^{8}$ & $\begin{array}{c}\text { DATE } \\
\text { de } \\
\text { G. SIMPSON }{ }^{\theta}\end{array}$ \\
\hline 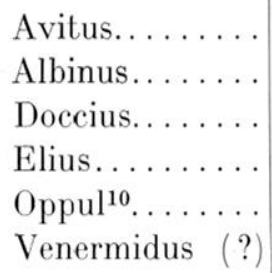 & $\begin{array}{l}1 \\
1 \\
2 \\
2 \\
2 \\
3\end{array}$ & $\begin{array}{l}\text { AVITVS } \\
\text { ALBINVS } \\
\text { DOCGIVSF } \\
\text { ELIMAN + } \\
\text { (voir fig. 3) } \\
\text { VIINIIRMIDIM }\end{array}$ & $\begin{array}{l}\text { Vespasien-Trajan } \\
\text { Trajan-Antonin } \\
\text { Domitien-Antonin } \\
\text { Hadrien-Antonin } \\
\text { Trajan } \\
\text { Trajan-Hadrien (?) }\end{array}$ & $120-160$ \\
\hline
\end{tabular}

La pâle est trìs blanche, écrue, grise ou à peine rosée, avec quelques traces de dégraissant (poterie écrasée). bien cuite, sans avoir la dureté de celle des vases rouges, sigillés ou lisses.

Le décor est peint directement sur la terre, habitucllement bien lissée, avec de l'ocre en général assez clair ${ }^{7}$. Ie rebord est décoré, à l'extérieur et très rarement à l'intérieur, de traits sensible-

mitres : 7 (3 ex.), 7,2 (2 ex.), 9 (5 ex.) et 10 (8 ex.) ; profil rare : fig. $2, n^{\circ} 2$. Plusicurs sont percés dans le centre à coups de poincon (cf. supports du Pont-des-Remes, dans G. Cunxwr et G. Gaudrox, La céramique sigillée l'Argonne des $I I^{\mathrm{e}}$ el $I I I^{\mathrm{e}}$ siècles, VI $I^{\mathrm{e}}$ supplément à Gallia, 1955 , p. 100 et fig. 44).

(7) Le produit employé semble noir avant cuisson. Sur les vases brisés avant de repasser au four, la couleur peinte a été délavée par un long séjour dans le sol.

(8) F. Oswald, Index of Pollers' Slamps on Terra sigillala, Margidunum, East Bridgford, 1931.

(9) J. A. Stanfield et Grace Simpson, Cienlral Gaulish Pollers, Oxford, University Press, 1958.

(10) Leurs estampilles sont imprimées sur des vases décorés des mêmes oves et des mêmes motifs. I.es trois dernières lettres de la marque que l'on a lue OPPYI. sont incertaines, encore que très bien dessinées. On pourrail lire aussi OPI'VA. La signature de ce potier est très friquente à Toulon. Les fouilles de cette officine montrent qu'il est plus tardif que ne l'admet Oswald, ou qu'il a continué son activité plus longtemps qu'il ne le pense. ment parallèles plus ou moins épais tracés de liaut en bas au pinceau. Sur quelques tessons, les traits sont interrompus
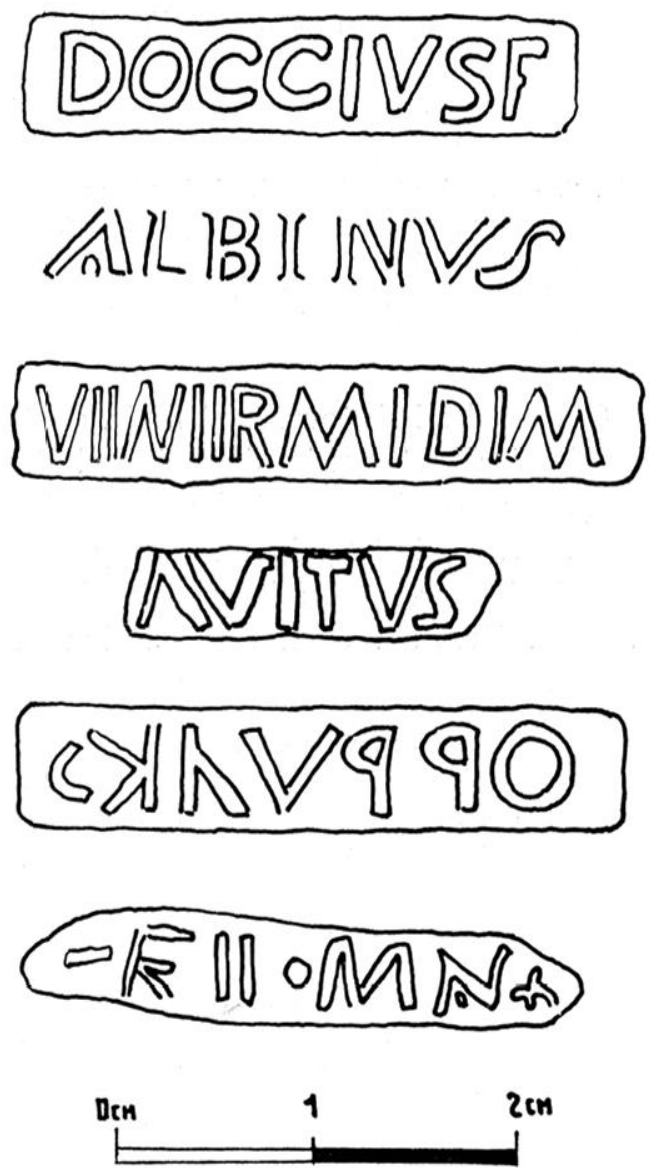

Fig. 3. - Jistampilles relevées sur les rehords des vases peints. (Dessins $I I$. Ver(el). 


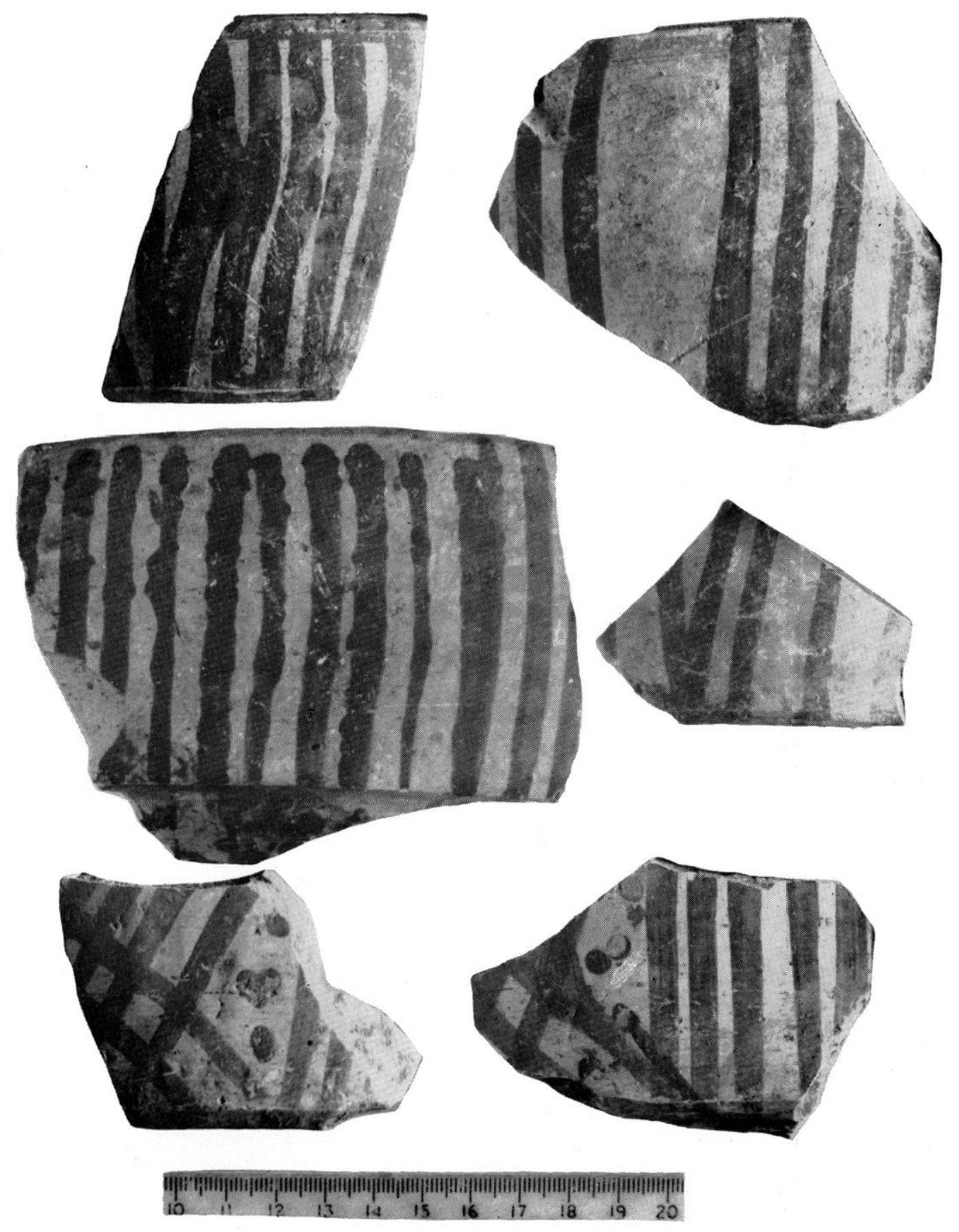

IFig. 4. - De hant en bas: différents rebords peints. En bas, croisillons et points. (Photos II. Vertel) 
par une zone de croisillons ; sur d'autres, quelques traits sont remplacés par des pointillés. Parfois la peinture, trop épaisse, a fait des bavures (fig. 4), Tout le reste du vase, intérieur, extérieur, pied compris, est marbré de la même ocre, parfois hâtivement, comme si on l'avait tamponné avec un gros pinceau ou une éponge. La peinture bien cuite résiste au lavage.

Nous avons relevé onze signatures, toujours imprimées sur le rebord du bol, a l'extéricur, en oblique. Elles sont identiques à celles de la céramique sigillée trouvée en même temps. Il est possible que d'autres potiers, dont les marques ont été recueillies dans le même ensemble, aient fabriqué aussi ce genre de vase (fig. 3).

L'étude de la céramique décorée qu'ont fabriquée ces potiers à Toulon-sur-Allier montre qu'ils sont contemporains. Il semble que l'on puisse dater cet ensemble des années 110-130 après J.-G. ${ }^{11}$.

Cette céramique n'a pas été souvent signalée en Gaule et nous serions heureux si l'on nous en indiquait l'existence dans les musées ou la découverte dans les fouilles, pour préciser sa durée et sa diffusion $^{12}$.

(11) D'après Oswald, la période où l'activité de ces potiers coïncide se situe entre les années 110 et 130. Miss Grace Simpson (ouvrage cilé) ne signale qu'un de ces potiers, Avilus, et porte son activité aux années 120-160. Mais il y eut peut-être plusieurs potiers de ce nom, qui n'était pas rare. Oswald mentionne 4 Avilus: le premier a I.a Graufesenque, le second à I.ezoux, le troisième à Rheinzabern, le quatrième à Eschweilerhof. İ. Driont, L'alelier de Salto, dans Mémoires de l'Académie nalionale de Melz, CXVII1c annee, $11^{\mathrm{e}}$ série, XVII, 1948, p. 95-127, et précisément p. 98 , en mentionne un à Chémery-Faulquemont, lequel a fabriqué seulement de petites tasses coniques ou hémisphériques, à l'exclusion d'autres formes lisses.

(12) On peut voir au Musée de Saint-Pèresous-Vézelay (Yonne) quelques tessons de cette
Parmi les officines du Centre de la Gaule, Toulon n'a pas le monopole de ce décor ni de cette forme; on a en effet trouvé des rebords à peine différents à Vichy et une peinture semblable à Lezoux ${ }^{13}$. Le pied évidé rappelle asse\% celui des vases gallo-romains précoces de Gergovie ${ }^{14}$, mais le profil du rebord et de la panse est proche de celui du vase I)ragendorff 45) à déversoir en mufle de lion ${ }^{15}$ (fig. 1 et 2 ).

Ce décor peint ne continue pas la tradition de celui des vases de la Tène III, qui a dû cependant se prolonger assez lardivement dans celte région ${ }^{16}$. C'est en Germanic que l'on peut trouver la mème ornementation à l'éponge. Le fond

céramique qui proviennent des fouilles des Fontaines-Salces.

(13) On trouve le même genre de rebord dans les vitrines du Vusie de Vichy, mais les traits de pinceau sont moins larges. Au Musée de Saint-Germain-en-Laye, des rebords provenant de Vichy portent une gorge marquée sous le décrochement.

(14) " A Gergrovie... le pied tourné est souvent remplacé par une espèce de pied en creux, de forme conique, décoré de rainures concentriques plus ou moins accentuées" (J.-J. HATT, Ĺssai d'une comparaison enlre la céramique cellique d'Aulnat-Sud el la céramique gallo-romaine précoce de Gergnvie, dans Bulletin historique el scientifique de l'Auvergne, no 528, 1954, fig. XVII, $\left.n^{\circ} 9,11,14\right)$. I a tradition celtique semble vivace a Toulon, dans la fabrication d'assiettes peintes comme dans certains décors sigillés particuliers.

(15) Mêlés à la masse des tessons de sigillée et de poterie peinte, plusieurs appliques et fragments de rebords de ce type de vase ont été trouvés à Toulon dans la même campagne de fouilles. Comme les vases peints, ces bols se brisent souvent sous le rebord.

(16) Les vases de la Tène II, plus artistiques, "apparaissent encore dans les nécropoles des preniers temps de l'époque impériale "(DécneI.ETTE, Mamuel, IV, p. 999); en note, l'auteur mentionne la decouverte de ces vases dans des niveaux des $11^{\mathrm{C}}$ et $11^{\mathrm{e}}$ s., mais donne cette indication comme peu sùre. On connaît de la sigillée marbre à lat Graufesenrue, mais d'un travail très fin et tont different. 

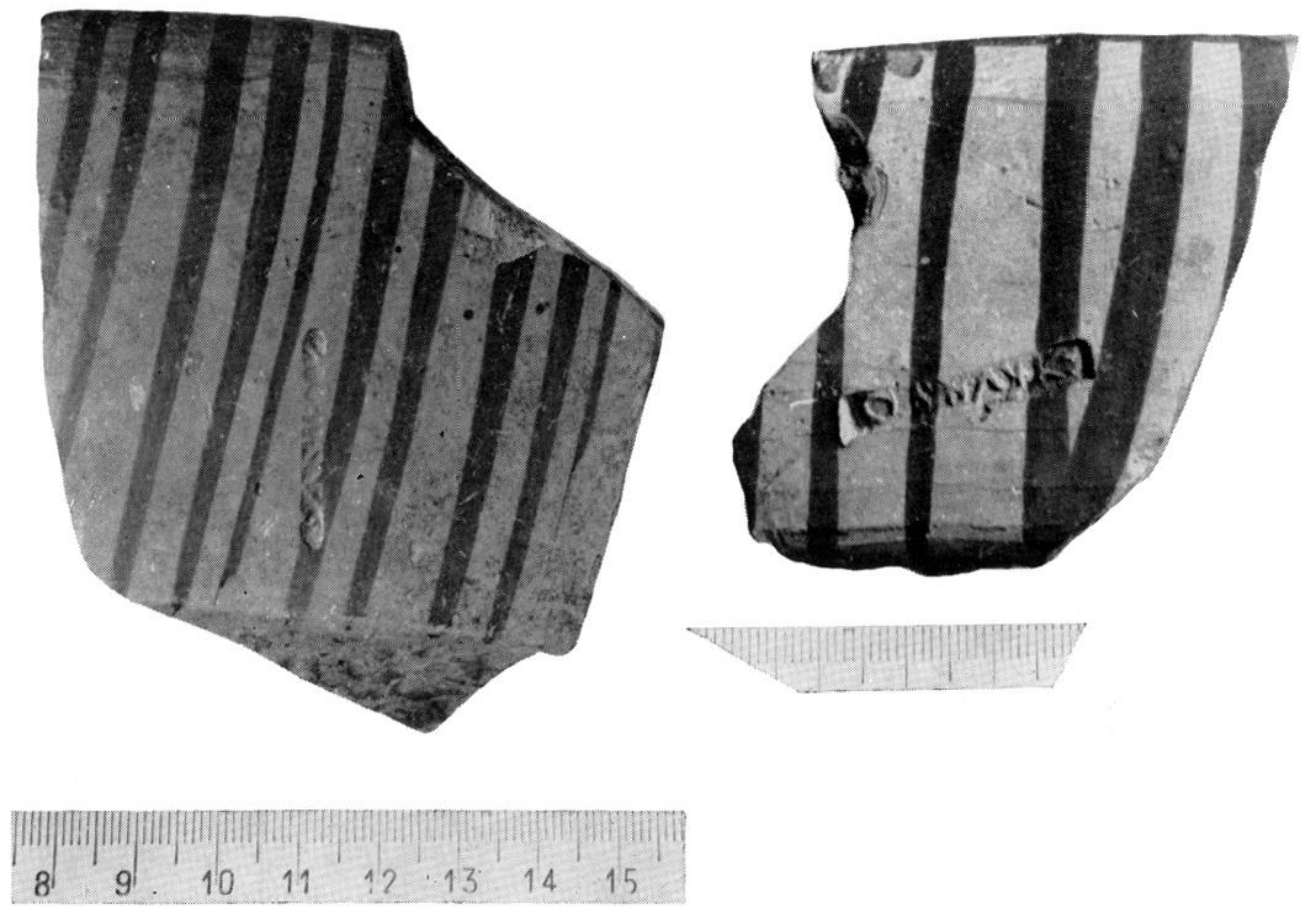

Figr. 5. - De gauche à droile: rebord signé AIBINVS, rehord signe OPPII.

clair, la peinture et la manière dont elle est appliquée, sont communs aux vases de Toulon et à ceux qu'E. Gose appelle la céramique marbrée de la première période, datée du dernier tiers du ${ }^{\text {er }}$ siècle et $d u$ premier tiers du ${ }_{11}{ }^{\mathrm{e}}{ }^{7}$. Or il est très possible qu'il $\mathrm{y}$ ait eu des points communs entre les oflicines du centre de la Gaule et celles de Germanie, par l'intermédiaire des légions romaines. En effet, la VIIIe Légion Auguste fut cantonnée à Néris en 70 après J.-C. Elle y eut três probablement des fabriques, puisqu'elle $\mathrm{y}$ laissa des tuiles estampillées ${ }^{18}$ et une

(17) Erich Gose, Gefässiypen der römischen Keramik im Rheinland, Rheinisches Landesmuseum, Bonn, 1950, p. 21 et suiv, et pl. XVIIXVIII.

(18) Léon Renier, dans Comples rendus de l'Académie des inscriplions et belles-lellres, 1872, p. 414. L'auteur démontre que la présence de la VII Ie Jégion à Néris a été motivée par la révolte antéfixe caractéristique ${ }^{19}$. Ses potiers eurent peut-être alors des rapports avec ceux de la vallée de l'Allier, peu éloignée, dont certaines oflicines étaient déjà actives à cette époque. Celte troupe fut ensuite envoyée en garnison à Argen-

du Batave Civilis, mais cette légion ne participa vraisemblablement pas à la guerre et fut laissée dans la Gaule comme arrière-garde.

(19) J. DÉchelette, Une anléfice de la Intitième Légion découverte à Véris, dans Comples rendus de l'tcademie des inscriptions el belleslellres, 1905, p. 597-602 : "Ces antéfixes ornées etaient evidemment façonnées avec des moules, comme les vases sigillés... Nous croyons... que les antefixes de Gaule sont de fabrication locale, car elles different de celles des pays classiques et présentent tous les caractères de l'art galloromain". L'existence d'une oflicine de potiers à Néris n'est pas encore prouvée et le Musée de cette ville ne conserve aucun tesson de vase peint. Mais il n'y eut jamais de fouilles sérieuses, et beaucoup de trouvailles fortuites ont été disperseies. 


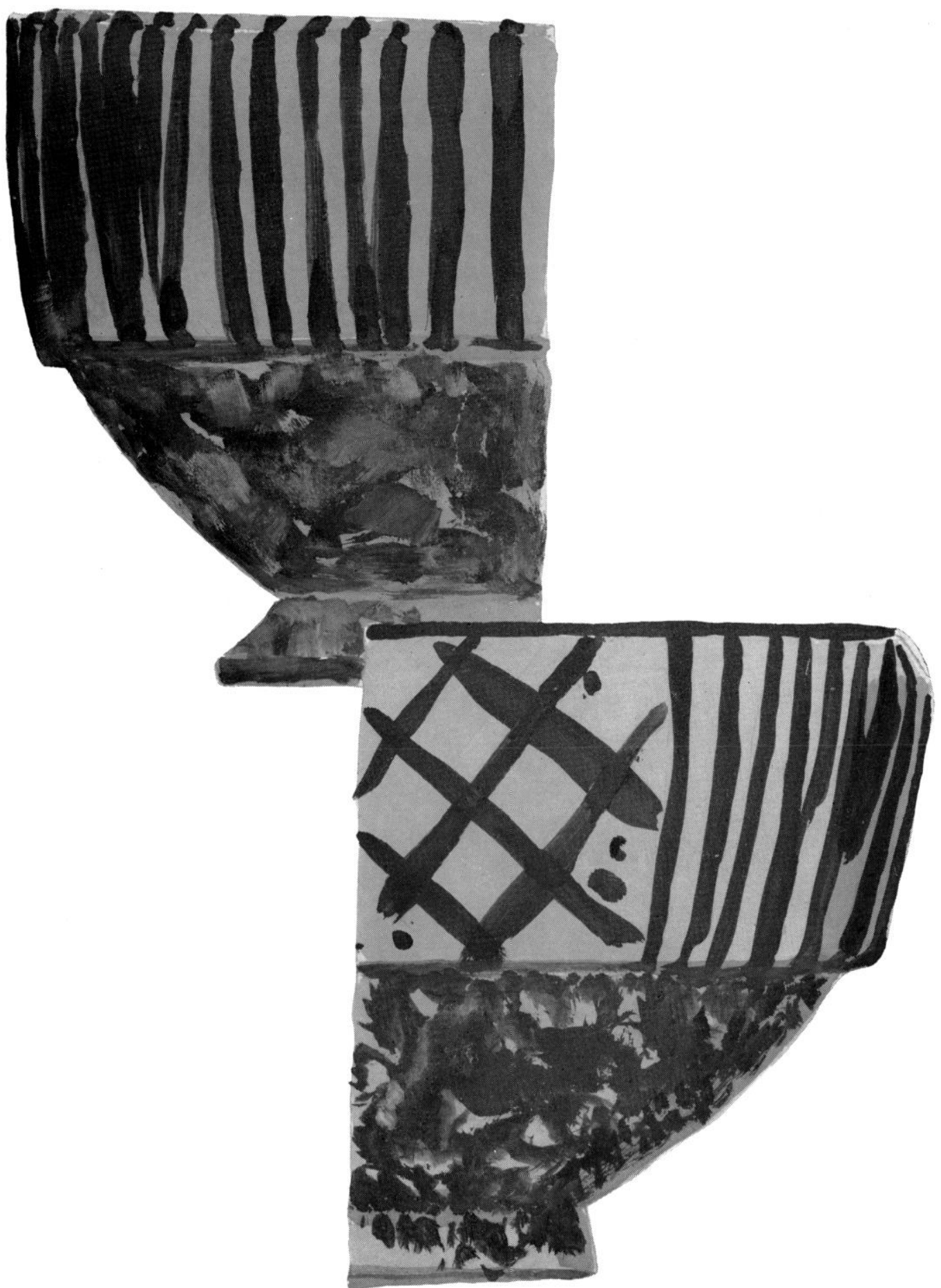

Fig. 6. - Keconstitutions de vases peints de l'oflicine de Toulon-sur-Allier (Allier). (Aquarelles de M. Brugnaud). 
torale ${ }^{20}$, où l'on a découvert récemment le dépotoir d'un atelier céramique légionnaire qui pourrait lui avoir apparlenu ${ }^{21}$. Il est intéressant de relever que la vaisselle militaire, et notamment celle de cette oflicine, comporte des vases peints. Mais les deux publications sur ce sujet, qui nous semblent les plus completes -

(20) "Après la guerre contre Saturninus, la Gaule étant pacifiée, cette légion fut envoyic dans le camp de Strasbourg " (J. Déchliletre, oul. cil., p. 602). On peut remar(uer, dans un ensemble de céramique très homogéne trouvé à Strasbourg, un fond de vase ovoidde en terre blanche peint en ocre rouge, par bandes verticales ... "avec un petil pincean on une plune" nous écrit M. IIatt ... et une estampille de la VIII e l.égion sur une tuile (J.-J. IIATT, Nouvelles découvertes romaines en Alsace, dans Cahiers d'archéologie et d'hisloire d'Alsace, 1948, p. 160182, pl. III, tesson $n^{\circ} 11$ ). Dans le même lot on peut noter des fragments de vases en terre blanche de la technique de Saint-Rémy-enRollat (Allier) : "morceau en terre blanche, couvert d'un vernis vert à base de plomb et qui porte encore la trace d'un relief (jambe et partie postérieure d'une figure de femme assise)" (ibid., p. 168 et pl. II, no 17). Peut-être est-il possible de rapprocher cette importation et l'existence d'un valeludinarium à Néris pendant de nombreuses années après le départ de la VIIIe Légion.

(21) J.-J. Hatr, dans Bulleiin de la Socielé nationale des antiquaires de France, 1952-1953, p. 170-171 et ID., Les fouilles de Strusbourg en 1953 el 1954. Découcerte d'un depoloir de céramique (Gallia, XII, 1954, p. 323-343). Pour les tuiles : Yowat, Les luiles de la légion VIII Augusla, à Strasbourg el à Viviers (Ardèche), dans Bullelin épigraphique de la Gaule, 1883, p. 303. colle d'Elisabeth Ettlinger sur Vindonissa et celle de J.-J. IIatt sur Strasbourg'22 ne présentent pas la forme que nous avons étudiée. Frich Gose ne l'a pas relevée non plus ${ }^{23}$. Il ne s'agit certainement pas de la même céramique, mais l'éventualité d'une influence technique des céramistes légionnaires sur ceux de l'Allier n'est pas exclue. Peut-être avons-nous là une production tout à-fait typique des ollicines de la Gaule centrale; peut-être ces différences s'expliquent-elles par une évolution divergente au cours de cinquante années, laquelle aurait abouti des deux còtés à des produits originaux.

Nous croyons en tous cas que ce rapprochement méritait d'être signalé. Espérons que des découvertes et des études ultérieures permettront d'éclaircir ce problème $^{24}$.

Hugues Vertet.

(22) J.-J. IIATt, arlicle cilé; E. ETthinger; IBömische Keramik aus dem Schutthügel von Vindonissa, Bâle, 1952, p. 57 et suiv.; ID., I.egionary Pollery from Vindonissa, dans Journal of Roman Studies, 1951, p. 105-111. Cette archéologue nous écrit : " Ich habe jedoch den Eindruck, dass essich bei Ihrer Keramik aus Toulon-surAllier um etwas anderes handelt als in Vindonissa ". Fffectivement les formes publiées ne portent ni traits au pinceau ni décor à l'éponge.

(23) E. Gose, ouvrage cilé, p. 21 : " die marmorierte Keramik enthält fast nur Trinkgeschirr, Napfe, Kruge und Kannen ".

(24) Nous remercions beaucoup IIme Elisabeth Ettlinger et M. J.-J. IIatt des comparaisons de céramique auxquelles ils ont bien voulu procéder et des suggestions qu'ils nous ont faites. 